\title{
Effect of Neuro-Linguistic Programming on Mental Toughness in Veteran and Disabled Athletes
}

\section{ART ICLE INF O}

\section{Article Type}

Original Research

\section{Authors}

Akbarzade B. ${ }^{1} M S c$,

Ahar S.* Msc,

Dadashzade $\mathrm{M}^{2} \mathrm{MSc}$

How to cite this article
Akbarzade B, Ahar S, Dadashza-
de M. Effect of Neuro-Linguistic
Programming on Mental Tough-
ness in Veteran and Disabled At-
hletes. Iranian Journal of War
\& Public Health. 2017;9(2):61-66.

*Motor Behavior Department, Physical Education \& Sport Sciences Faculty, Kharazmi University, Tehran, Iran

${ }^{1}$ Sport Psychology Department, Physical Education \& Sport Sciences Faculty, Allameh Tabataba'i University, Tehran, Iran

${ }^{2}$ Evaluating \& Measuring Department, Psychology \& Educational Faculty, Allameh Tabataba'i University, Tehran, Iran

\section{Correspondence}

Address: Faculty of Physical Education and Sport Sciences, Kharazmi University, Shahid Keshvari Stadium, Mirdamad Street, Tehran. Postal Code: 1464785581

Phone: +98 (21) 44118632

Fax: +98 (21) 44118629 saeid.ahar@gmail.com

\section{Article History}

Received: November 14, 2016

Accepted: January 31, 2017

ePublished: April 24, 2017

\section{A B S T R A C T}

Aims Any success in sport contests depends on mental preparation, alongside the physical and technical readiness, in the veteran and disabled athletes. The aim of the study was to investigate the effectiveness of the neuro-linguistic programming strategies training courses on the mental toughness in the veteran and disabled athletes.

Materials \& Methods In the controlled pretest-posttest semi-experimental study, 30 male veteran and disabled athletes with physical-motor disabilities were studied in Tabriz in 2016. The subjects, selected via available sampling method from GFT Sitting Volleyball Team, were randomly divided into two groups including experimental $(n=15$ persons) and control $(n=15$ persons) groups. Data was collected by Sheard et al. Questionnaire for mental toughness. After the pretest, sixteen 30-minute training sessions of neuro-linguistic programming strategies were conducted in experimental group. Data was analyzed using multivariate ANCOVA. Findings At the posttest stage, the mean score of mental toughness in experimental group was significantly higher than control group $(p=0.001)$. The mean scores of confidence $(p=0.026)$ and control $(\mathrm{p}=0.001)$ in experimental group were significantly higher than control group. However, the groups were not significantly different in sustainability $(\mathrm{p}=0.673)$.

Conclusion Improving their sport activities, teaching the neuro-linguistic programming strategies affects the mental toughness in the veteran and disabled athletes.

Keywords Mental Toughness; Confidence; Veterans; Disabled Persons, Thinking Skills

\section{I T A T I O N L I NKS}

[1] Study for determining laterality in children with motor disabilities in adapted ... [2] The quality of care and support (QOCS) for people with disability scale: development and ... [3] The relationship between self-esteem and sexual selfconcept in people with ... [4] Goodling MD. Sport psychology: An undeveloped discipline from among ... [5] Comparing the mental health of the athletic and non- athletic ... [6] Progress toward construct validation of the sports mental ... [7] Coaches' views of mental toughness and how it ... [8] How the best of the best get better and ... [9] Anxiety, expertise, and visual search strategy ... [10] Sports slump busting: 10 steps to mental toughness and ... [11] Evaluation of a mental toughness training program for youth-aged Australian footballers ... [12] What is this thing called mental toughness? An investigation of elite sport ... [13] Mind over matter: The development of the mental toughness ... [14] A review and conceptual re-examination of mental toughness: Implications for future ... [15] Mental toughness: Trait or developmental capability? effectiveness ... [16] Mental toughness, optimism, pessimism, and coping among ... [17] A behavioural genetic study of ... [18] Effect of a psychological skills training program on swimming performance and positive psychological [19] Examining therelationship between mentaltoughness and ... [20] Handbookofsport... [21] Neuro linguistic programming for ... [22] Neuro-linguistic programming and learning theory ... [23] Neuro-linguistic program: theory and Psychological [24] Using NLP in sport psychology; neuro-linguistic programming affects on boxer .. [25] Sport psychology and use of neuro ... [26] The Effect of Neuro-linguistic programming ... [27] Influence of mental training on the execution ... [28] Neurolinguistic programming based on the concept ... [29] psycho-educational programme for cricket players using ... [30] Psychometric properties and ... [31] Applied sport psychology: Personal growth to peak ... [32] The effect of NLP strategies training ... [33] Effectiveness of group cognitive behavioral therapy and group cognitive ... 
است[5]. يكى از متغيرهايى كه در تحقيقات روانشناسى ورزشى إسى

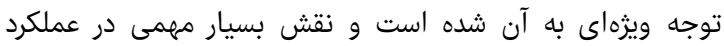

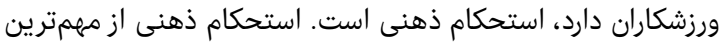
ويزّى هاى موثر در دستيابى به موفقيت ورزشى دانى در نظر گَرفته شده

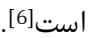

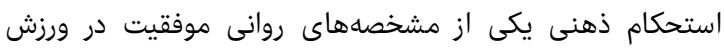

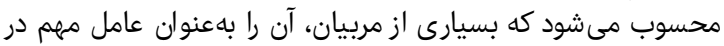

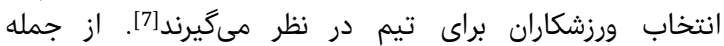

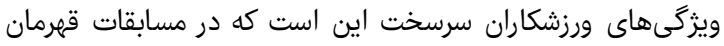

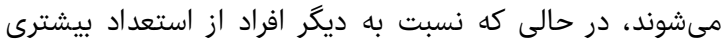

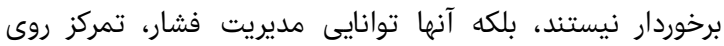

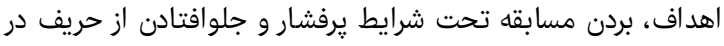

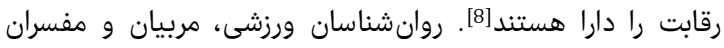

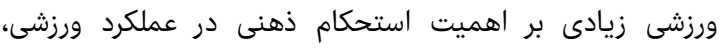

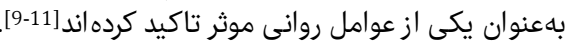

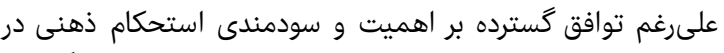

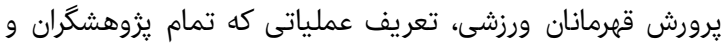

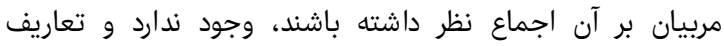

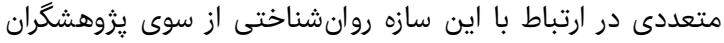

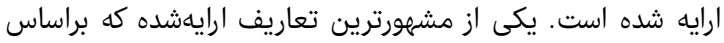

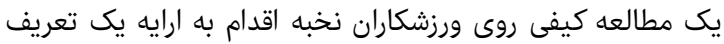

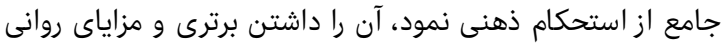

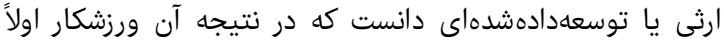

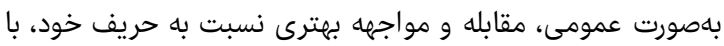

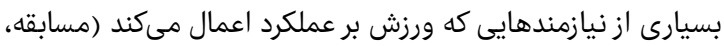

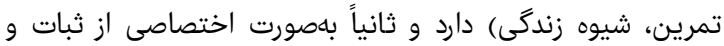

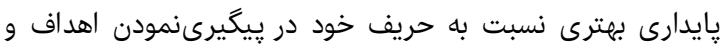

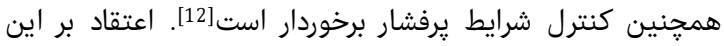

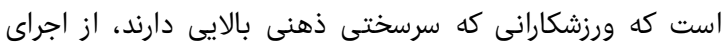

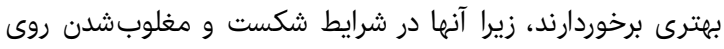

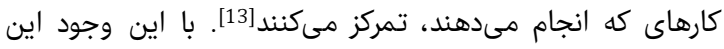

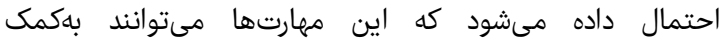

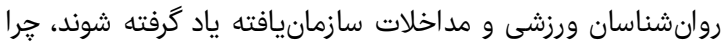

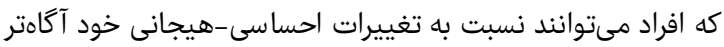

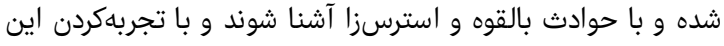
شرايط، از اين آكاهى براى ياسخ يا رفتار إنتار بهشكل موثرترى استفاده

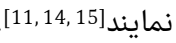
مرور مطالعات اوليه در زمينه استحكام ذهنى نشان ميددهد كه

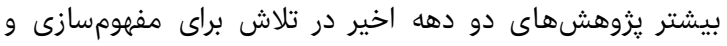

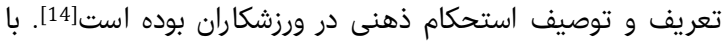

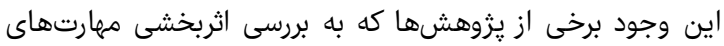

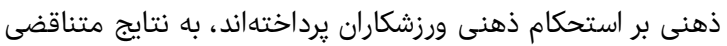

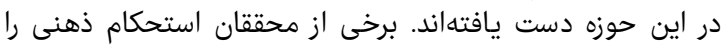

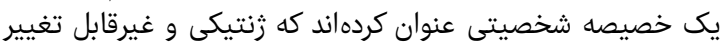

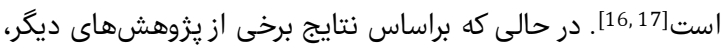

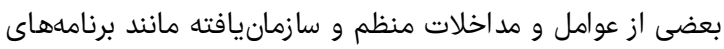

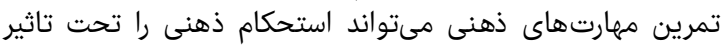

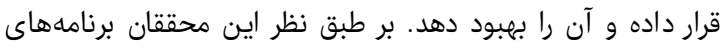
همهارتهاى ذهنى را مىتوان به ورزشكاران معلول آموزش داد-8,18]

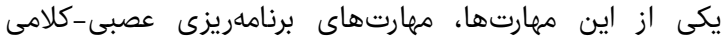

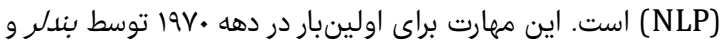

دوره 9، شماره r، بهار عوسا

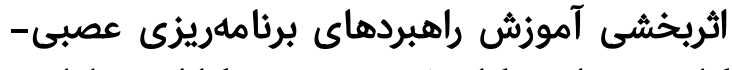

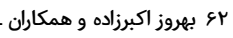

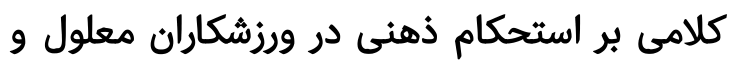
جانباز

MSc - بهروز اكبرزاده كروه روان شناسى ورزش، دانشكده تربيت بدنى و علوم ورزشى، دانشكاه علامه طباطبائى، تهران، ايران

MSc سعيد آهاري، تروه رفتار-حركتى، دانشكده تربيت بدنى و علوم ورزشى، دانشكاه خوارزمى، تهران، ايران

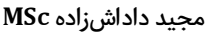

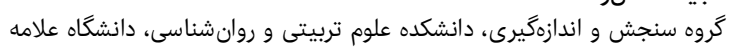
طباطبائى، تهران، ايران

קكيده

اهّداف: موفقيت در ميادين بين المللى ورزشى، در گرو آمادگى روانى ورزشكاران

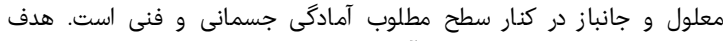

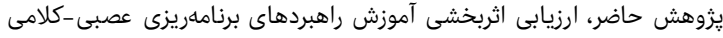

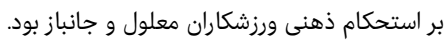

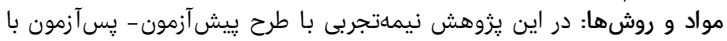

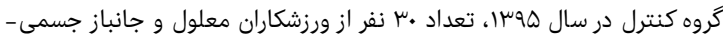

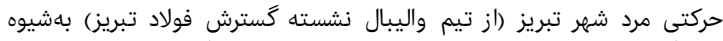

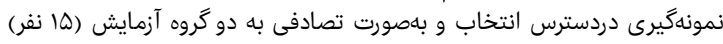

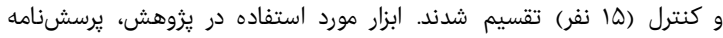

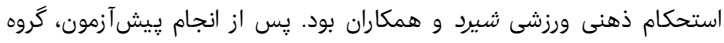

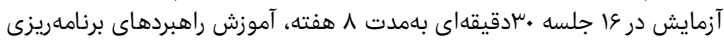

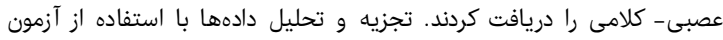

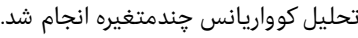

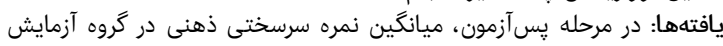

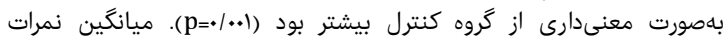

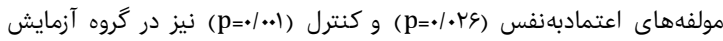

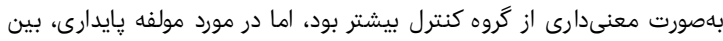

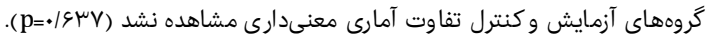

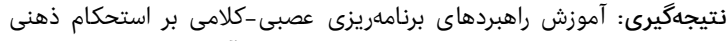

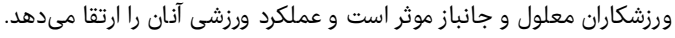

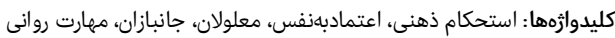

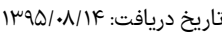

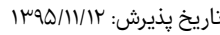

saeid.ahar@gmail.com : تويسنده مسئول:

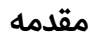

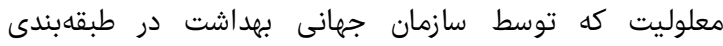

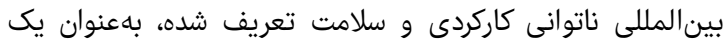

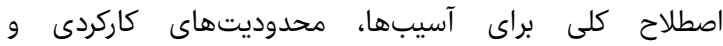

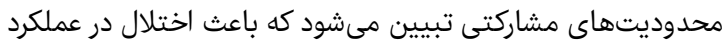

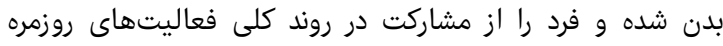

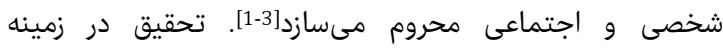

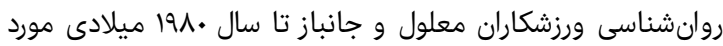

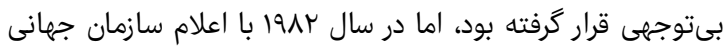

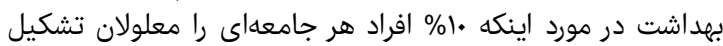

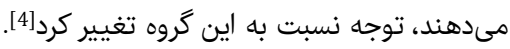

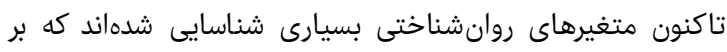

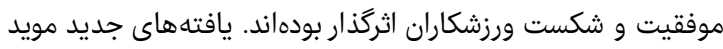

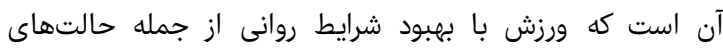

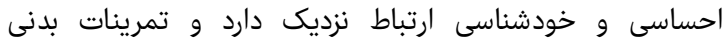

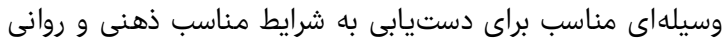


اثربخشى آهوزش راهبردهاى برنامهريزى عصبى-كلامى بر استحكام ذهنى در ورزشكاران معلول و جانباز سباء

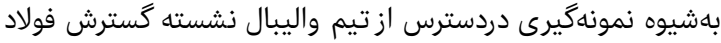

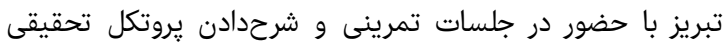

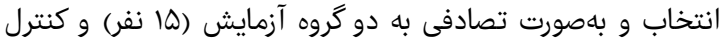

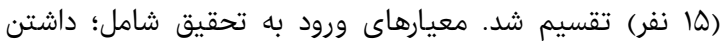

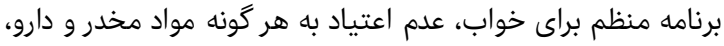

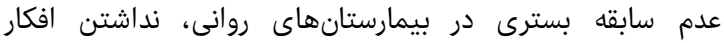

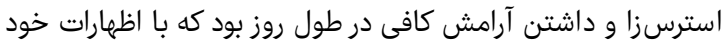

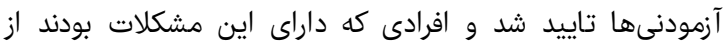

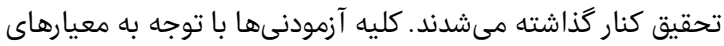

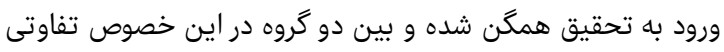

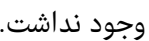

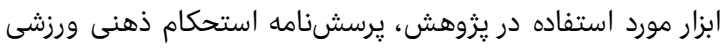

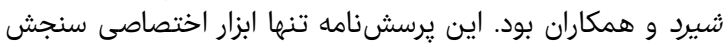

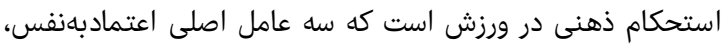

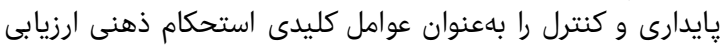

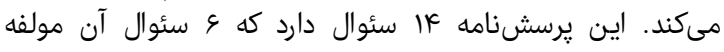

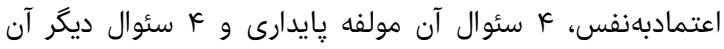

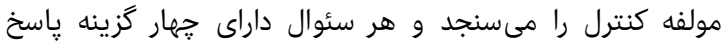

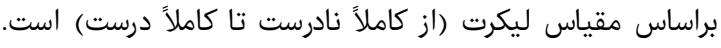

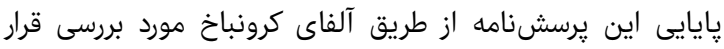

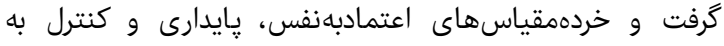

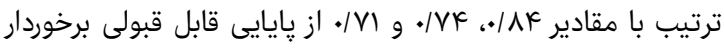

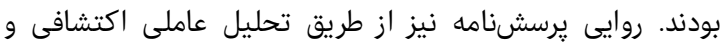

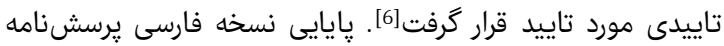

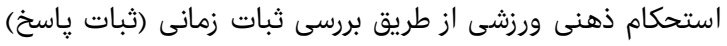

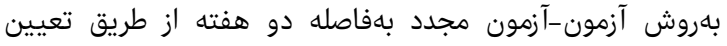

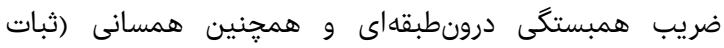
درونى) سئوالات از طريق آلفاى كرونباخ مورد بروسى و وريقي تاييد قرار

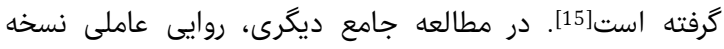

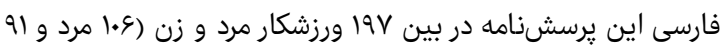

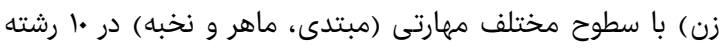

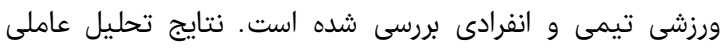

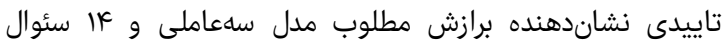
نسخه فارسى يرسش نامشامه استحكام ذهنى ورزشى بود [30].

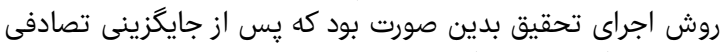

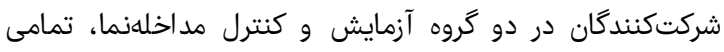

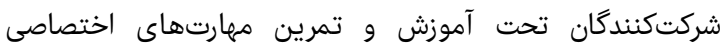

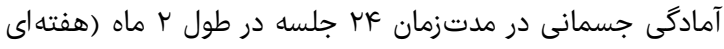

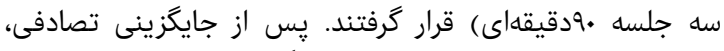

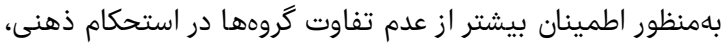

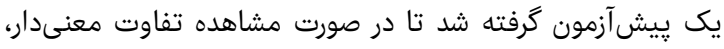

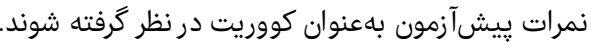

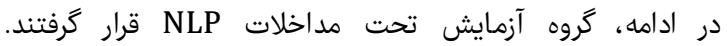

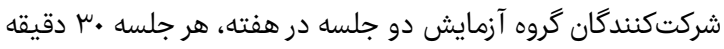

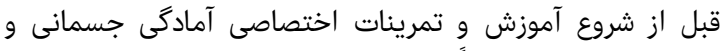

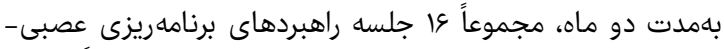

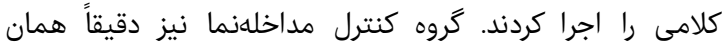

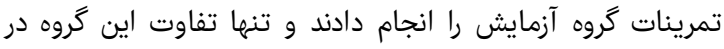

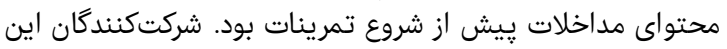

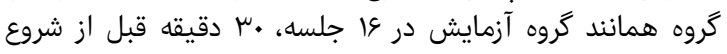

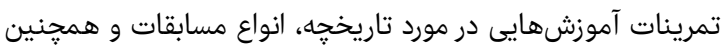
اطلاعاتى در مورد اهميتداشتن آمادگى جسمانى دردئ دريافت كردند

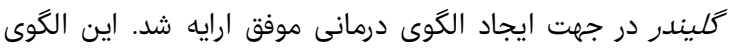

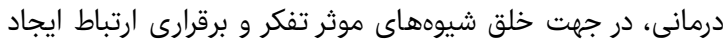

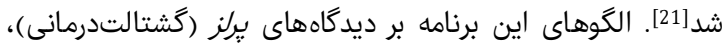

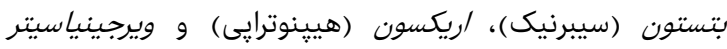

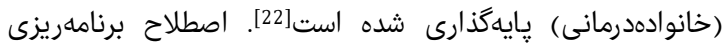

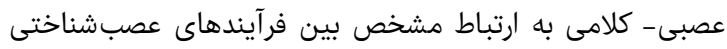

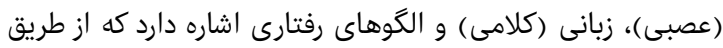

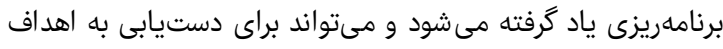

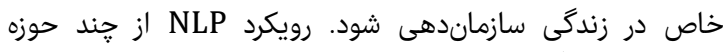

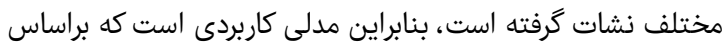

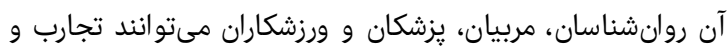

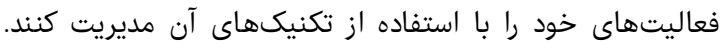

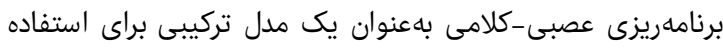

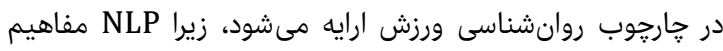

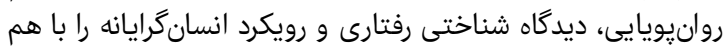

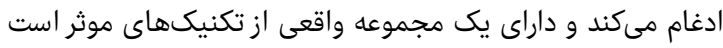

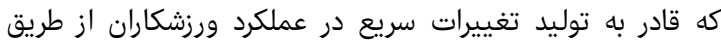

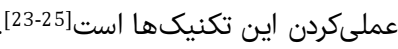

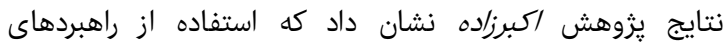

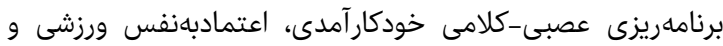

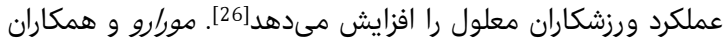

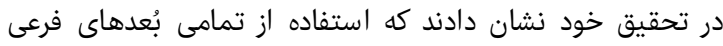

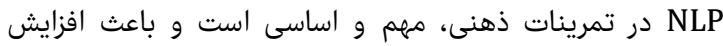

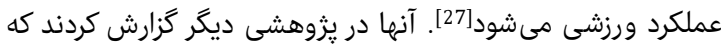

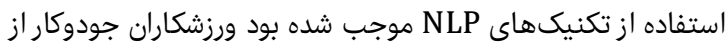

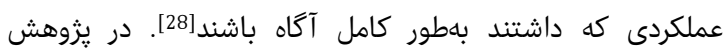

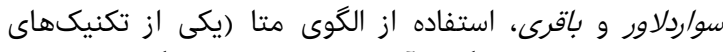

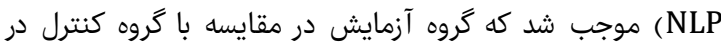

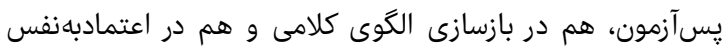

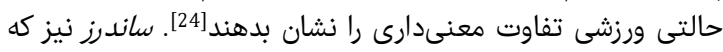

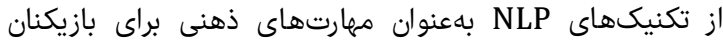

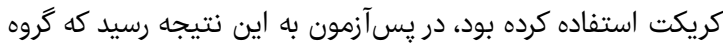

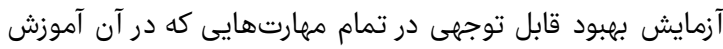

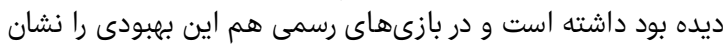

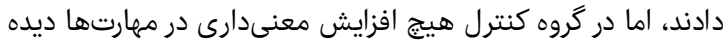

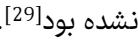

بيشتر مطالعات انجامشده ديدگاه مثبتى نسبت بـاه بهاه برنامهريزى

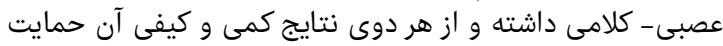

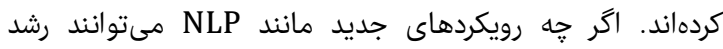

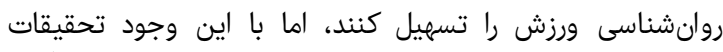

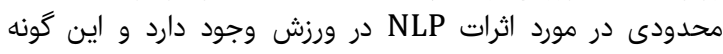

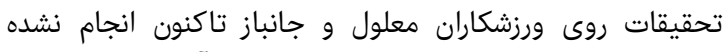

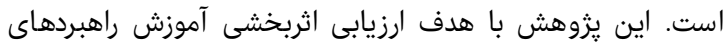

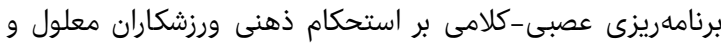

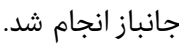

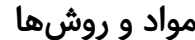

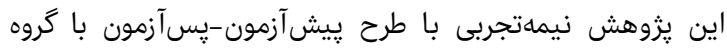

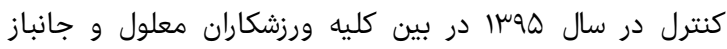

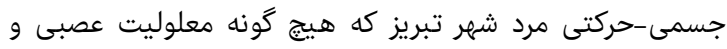
شناختى نداشتند، انجام شدر شرد نمونه مورد نظر با حجم •س نفر 


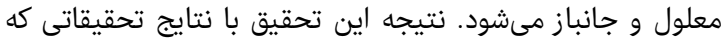

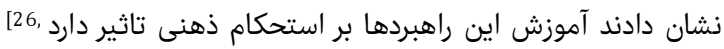

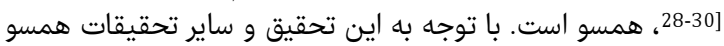

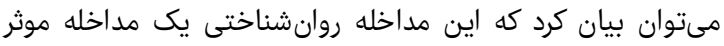

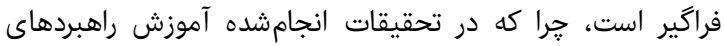

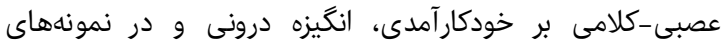

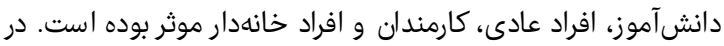

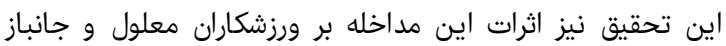
كزارش شد.

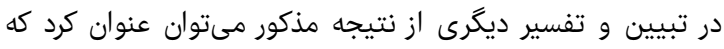

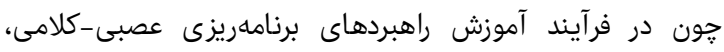

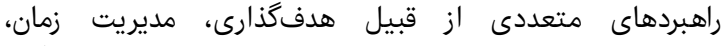

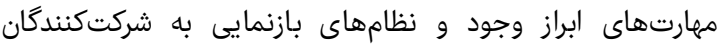

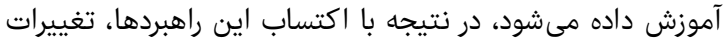

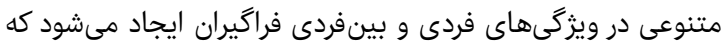

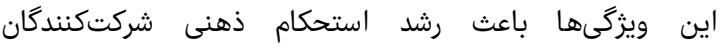

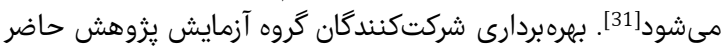

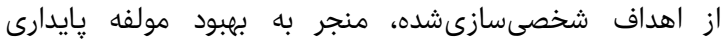

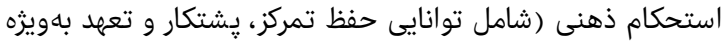

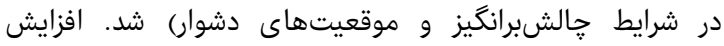

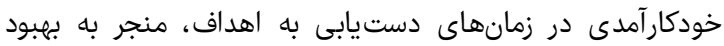

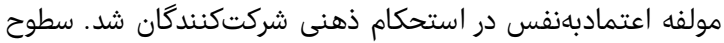

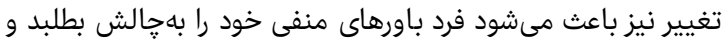

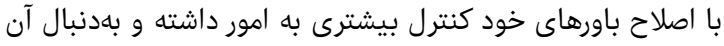

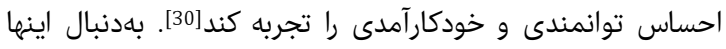

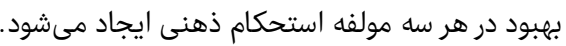

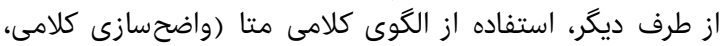

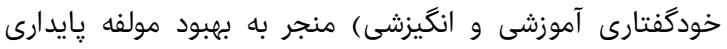

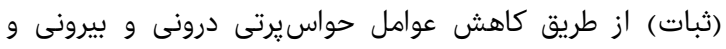

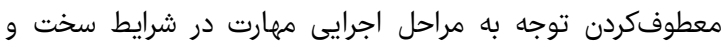

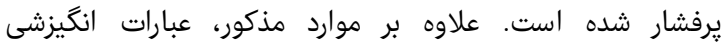

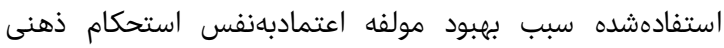

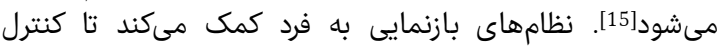

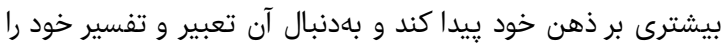

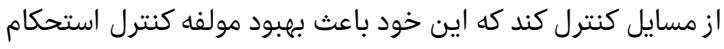

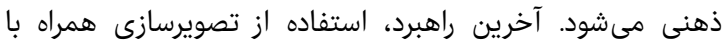

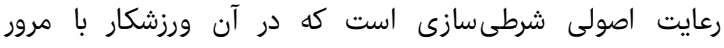

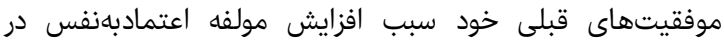

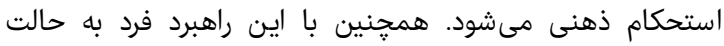

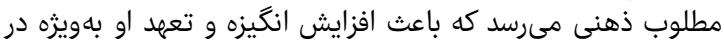
شرايط يرفشار و در نتيجه سبب بهاعب إزبود مولفه يايدارى استحكام ذهنى مى شود [26]. اما از سويى، نتايج تحقيق حاضر با ساير تحقيقات كه از اين مداخله

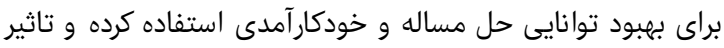

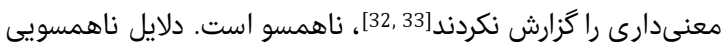

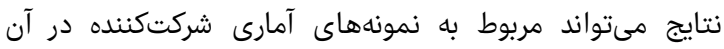

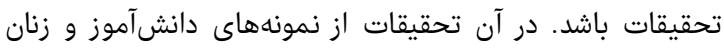

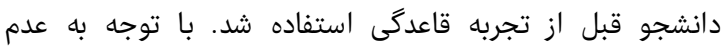

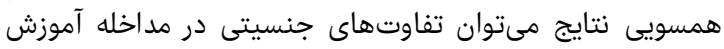

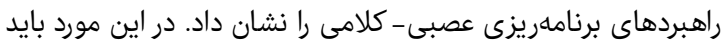

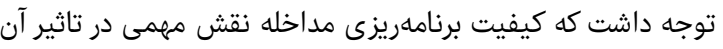

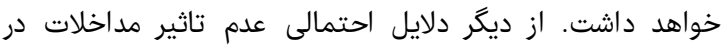

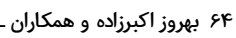

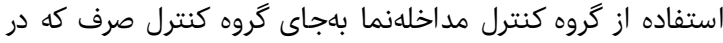

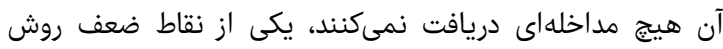

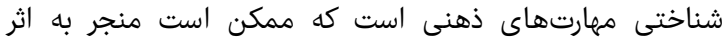

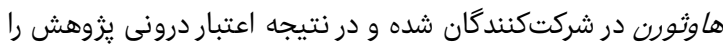

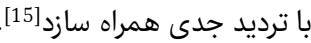

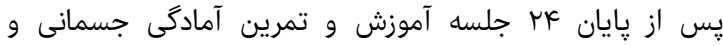

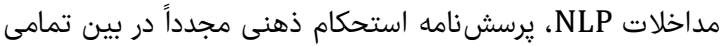

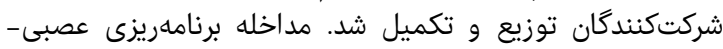

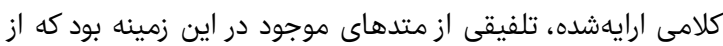

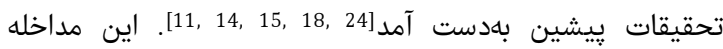

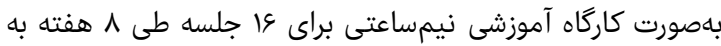

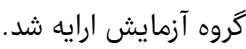

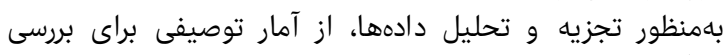

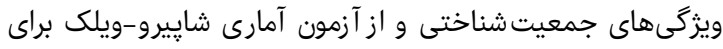

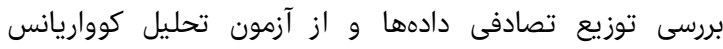

جندمتغيره براى بررسى اثربخشى مداخله ارايهشده استفاده شد.

يافتهها

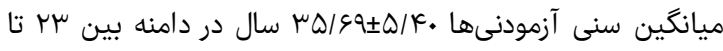

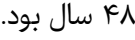

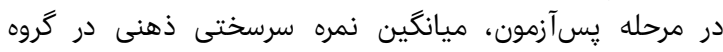

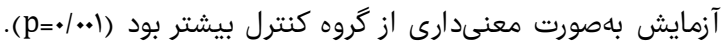

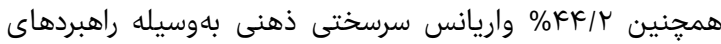

NLP

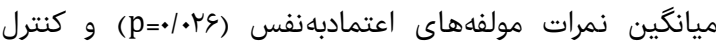

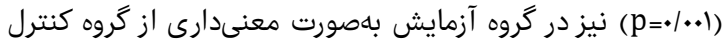

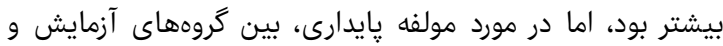

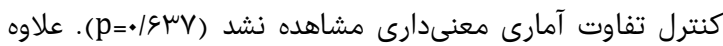

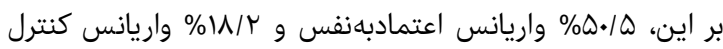
بهوسيله راهبردهاي NLP تبيين شد (جدول () ).

جدول () مقايسه ميانكين آمارى نمرات سرسختى ذهنى و مولفههاى آن، قبل و

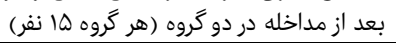

\begin{tabular}{|c|c|c|c|}
\hline گروه كنترل & گروه آزمايش & متغيرها & \\
\hline $\begin{array}{l}\mid Q / \zeta \cdot \pm 1 / / . \\
\mid V / r \cdot \pm \Psi / Q .\end{array}$ & $\begin{array}{l}1 \Delta / 1 \cdot \pm r / 1 . \\
r \mu / / \Delta \pm 1 / K .\end{array}$ & & بش برآزمتى ذهنى \\
\hline $\begin{array}{l}\mid \mu / r \Delta \pm \mu / q . \\
\mid r / \kappa \cdot \pm \mu / \kappa r\end{array}$ & $\begin{array}{l}\mid \mu / r \cdot \pm r / q . \\
\mid V / \mu \mu_{ \pm}+1 / q \Delta\end{array}$ & & يش \\
\hline $\begin{array}{l}Q / \Lambda \cdot \pm \mu / r . \\
1 \cdot / \cdot \pm r / \Lambda .\end{array}$ & $\begin{array}{l}1 \cdot / r \Delta \pm r / \Delta . \\
\| / / r \pm r / 1 / \mu\end{array}$ & & ش شَآزمون \\
\hline $\begin{array}{l}\| / 1 \cdot \pm r / k . \\
\| / \mu \mu \pm \Delta / \mu V\end{array}$ & $\begin{array}{l}1 . / r \Delta \pm 1 / \gamma . \\
\mid r / / \mu \pm \pm / \lambda \psi\end{array}$ & & شف كنترل \\
\hline
\end{tabular}

بحث

هدف از اجراى اين يزوهش، تعيين اثربخشى آموزش راهبردهاى

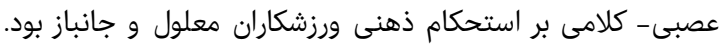

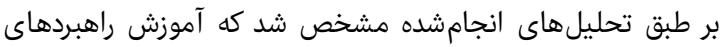

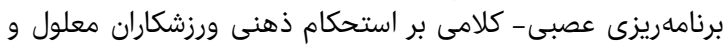

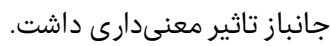

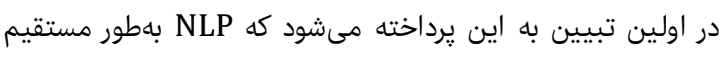
بهنوان مهارتهاى ذهنى باعث رشد استحكام ذهنى ورزشكاران 


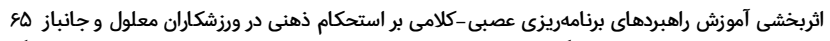

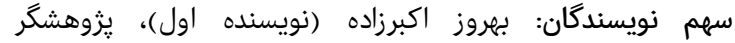

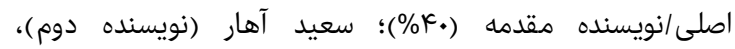

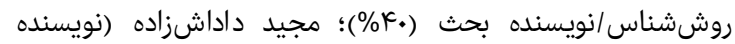

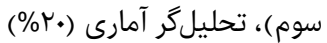

منابع مالى: منابع مورد نياز اين يزوهش إمش توسط محققان تامين شد.

منابع

1- Aurora U. Study for determining laterality in children with motor disabilities in adapted physical activities. Procedia Soc Behav Sci. 2014;117:646-52.

2- Lucas-Carrasco R, Eser E, Hao Y, McPherson KM, Green A, Kullmann L, et al. The quality of care and support (QOCS) for people with disability scale: development and psychometric properties. Res Dev Disabil. 2011;32(3):1212-25.

3- Salehi M, Tavakol HK, Shabani M, Ziaei T. The relationship between self-esteem and sexual self-concept in people with physical-motor disabilities. Iran Red Crescent Med J. 2015; 17(1):e25359.

4- Asken MJ, Goodling MD. Sport psychology: An undeveloped discipline from among the sport sciences for disabled athletes. Hum Kinet J. 1986;3(4):312-19.

5- Poursoltani Zarandi H, Rahmaninia F, Dejahang M, Mohaddes F. Comparing the mental health of the athletic and non- athletic physically- disabled people. Iran J Health Phys Act. 2011;2(1):6-10. [Persian]

6- Sheard M, Golby J, Van Wersch A. Progress toward construct validation of the sports mental toughness questionnaire (SMTQ). Eur J Psychol Assess. 2009;25(3):186-93.

7- Weinberg R, Butt J, Culp B. Coaches' views of mental toughness and how it is built. Int J Sport Exerc Psychol. 2011;9(2):156-72.

8- Jones G. How the best of the best get better and better. Harv Bus Rev. 2008;86(6):123-7.

9- Williams AM, Elliott D. Anxiety, expertise, and visual search strategy in karate. J Sport Exerc Psychol. 1999;21(4):362-75.

10- Goldberg AS. Sports slump busting: 10 steps to mental toughness and peak performance. Tamarac, Florida: Llumina Press; 2005.

11- Gucciardi DF, Gordon S, Dimmock JA. Evaluation of a mental toughness training program for youth-aged Australian footballers: II. a quantitative analysis. J Appl Sport Psychol. 2009;21(3):324-39.

12- Jones G. Hanton S, Connaughton D. What is this thing called mental toughness? An investigation of elite sport performers. J Appl Sport Psychol. 2002;14(3):205-18.

13- Madrigal L, Hamill S, Gill DL. Mind over matter: The development of the mental toughness scale (MTS). Sport Psychol. 2013;27(1):62-77.

14- Crust L. A review and conceptual re-examination of mental toughness: Implications for future researchers. Personal Individ Differ. 2008;45(7):576-83.

15- Motasharrei E, Abdoli B, Vaez Mousavi SMK, Farsi AR. Mental toughness: Trait or developmental capability? effectiveness evaluation of psychological skills training. Sport Psychol Stud. 2015;4(13):67-88. [Persian]

16- Nicholls AR, Polman RC, Levy AR, Backhouse SH. Mental toughness, optimism, pessimism, and coping among athletes. Personal Individ Differ. 2008;44(5):1182-92.

17- Horsburgh VA, Schermer JA, Veselka L, Vernon PA. A behavioural genetic study of mental toughness and personality. Personal Individ Differ. 2009;46(2):100-5.
تحقيقات ناهمسو مىتوان به زمان ناكافى و عدم برنامهريزى جامع

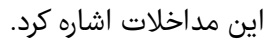

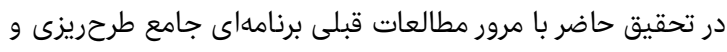

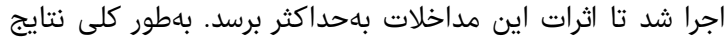

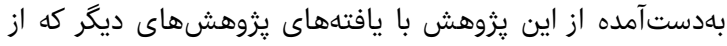

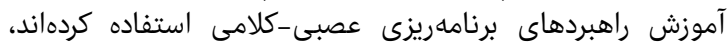

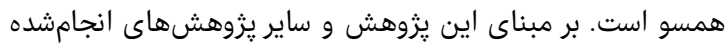

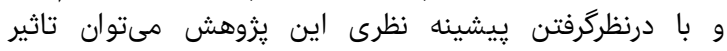

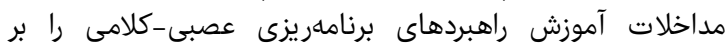

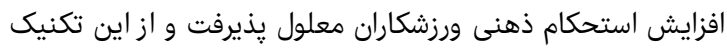

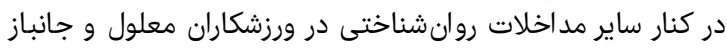

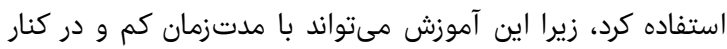

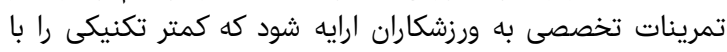

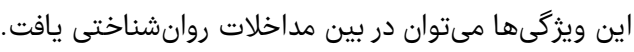

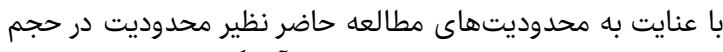

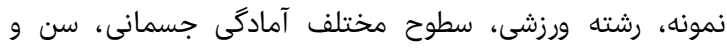

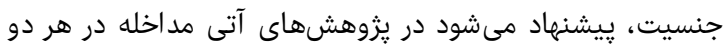

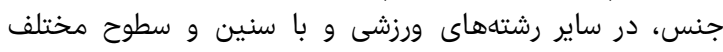

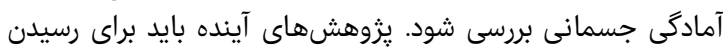

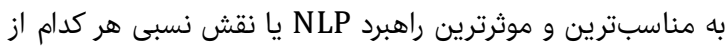

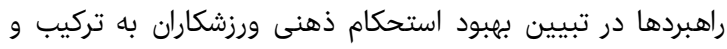

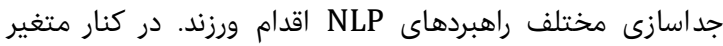

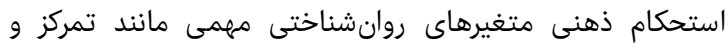

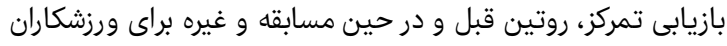

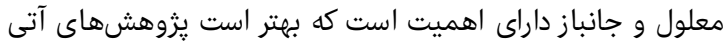

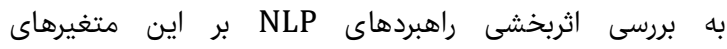

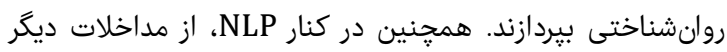

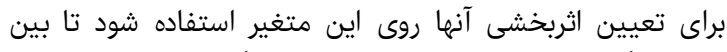

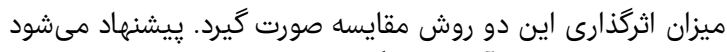

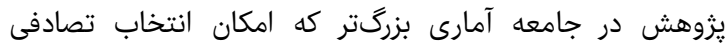

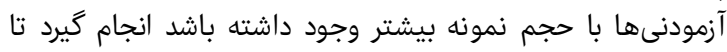

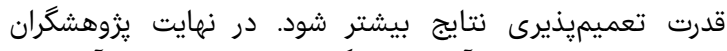

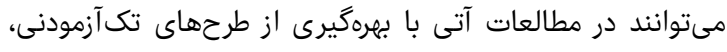

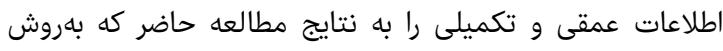
كروهمحور انجام شد، اضافه نمايند. تكميل

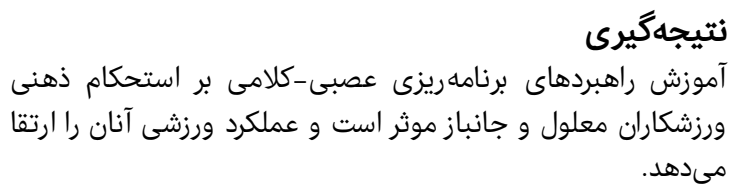

تشكر و قدردانى: بلرسم ادب از زحمات تيم يزوهش، رييس و دبير

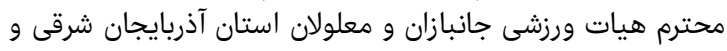

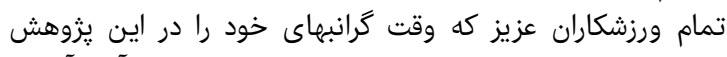

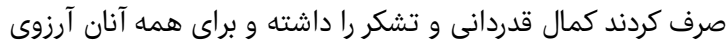

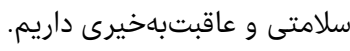

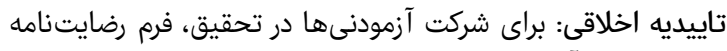

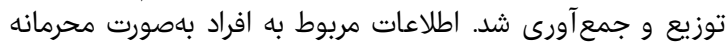

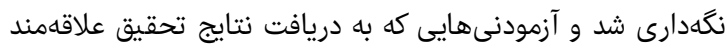

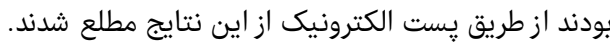

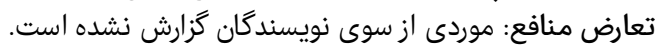

Volume 9, Issue 2, Spring 2017 
Tabataba'i University; 2016. [Persian]

27- Moraru CE, Radu LE, Grosu EF, Puni AR. Influence of mental training on the execution technique in rhythmic gymnastics. Glob J Humanit Soc Sci. 2015;1(1):26-34.

28- Grosu EF, Grosu VT, Preja CA, Iuliana BB. Neurolinguistic programming based on the concept of modelling. Procedia Soc Behav Sci. 2014;116:3693-9.

29- Saunders DE. A psycho-educational programme for cricket players using neuro-linguistic programming [Dissertation] Stellenbosch: University of Stellenbosch; 2009.

30- Kashani V, Shiri H, Manasei H. Psychometric properties and normalization of persian version of mental toughness scale (MTS). Res Univ Sport. 2015;7(2):83-98. [Persian]

31- Williams JM, Harris DV. Applied sport psychology: Personal growth to peak performance; 2nd edition. Mountain View, CA, US: Mayfield Publishing Co; 1993. pp. 285-305.

32-Zamini S, Hosseini Nasab SD, Hashemi T. The effect of NLP strategies training on self-efficacy and problem solving among girl students. J Psychol. 2009;13(3):25871. [Persian]

33- Moghtader L, Hasanzade R, Mirzaeian B, Dusti YA. Effectiveness of group cognitive behavioral therapy and group cognitive hypnotism on anxiety and depression in women with premenstrual syndrome. J Holist Nurs Midwifery. 2016;26(81):96-105. [Persian]

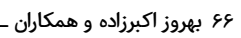

18- Sheard M, Golby J. Effect of a psychological skills training program on swimming performance and positive psychological development. Int J Sport Exerc Psychol. 2006;4(2):149-69.

19- Mattie P, Munroe-Chandler K. Examining the relationship between mental toughness and imagery use. J Appl Sport Psychol. 2012;24(2):144-56.

20- Tenenbaum G, Eklund RC. Handbook of sport psychology. 3rd edition. Hoboken, NJ: Wiley; 2007.

21- Harman RL, O'neill C. Neuro linguistic programming for counselors. J Couns Dev. 1981;59(7):449-53.

22- Tosey P, Mathison J. Neuro-linguistic programming and learning theory: A response. Curric J. 2003;14(3):371-88.

23- Savardelavar M. Neuro-linguistic program: theory and Psychological applications. Tehran: Arjmand Publication; 2010. [Persian]

24- Savardelavar $M$, Bagheri AH. Using NLP in sport psychology; neuro-linguistic programming affects on boxer state-sport confidence by using Meta-models method. Eur J Exp Biol. 2012;2(5):1922-7.

25- Lazarus J, Cohen R. Sport psychology and use of neuro linguistic programming (NLP) in sport. J Health Soc Environ Issues. 2009;10(1):5-12.

26- Akbarzadeh B. The Effect of Neuro-linguistic programming strategies on general self-efficacy, sport self-confidence and athletic performance of sitting volleyball team [Dissertation]. Tehran: Allameh 